\title{
Social and psychological assessment of women undergoing elective oocyte cryopreservation: A 7-year analysis
}

\author{
Victoria Vallejo ${ }^{1}$, Joseph A. Lee ${ }^{1 *}$, Lisa Schuman ${ }^{1}$, Georgia Witkin ${ }^{1,2}$, Enrique Cervantes ${ }^{1}$, \\ Benjamin Sandler ${ }^{1,2}$, Alan B. Copperman ${ }^{1,2}$ \\ ${ }^{1}$ Reproductive Medicine Associates of New York, New York, USA \\ ${ }^{2}$ Reproductive Endocrinology, Mount Sinai School of Medicine, New York, USA \\ Email: ${ }^{*}$ jlee@rmany.com
}

Received 21 November 2012; revised 23 December 2012; accepted 31 December 2012

\begin{abstract}
Our study focused on a retrospective analysis from 2004-2011 of patients considering elective oocyte cryopreservation (OC). We investigated the psychological and social aspects related to women who electively cryopreserve oocytes. Over seven years, consulted patients $(n=315)$ considering non-medical OC were interviewed by the staff therapists. Social, demographic, motivational impetus, psychological factors and local to national economy were analyzed in association with trends in elective OC. Patient disclosure, fertility assessment and receptivity to potential single motherhood were other aspects examined. Statistical analysis was performed with Student's t-test, Pearson's correlation and Chi-square analysis. Advanced technology, decreased age $(<35)$, anual per capita income, levels of follicular stimulant hormone (FSH) and basal antral follicular count (BAFC) were demonstrated to be the most influential factors of elective OC. The mean age of elective OC patients was $38.6 \pm$ 1.83 with nearly $80 \%$ of these patients disclosing their decisions either with family and/or friends. Clinical perception has increasingly improved the availability and efficacy of elective oocyte cryopreservation, albeit minimal publications have studied the social and epidemiological aspects of such patients. We identified these patients are often motivated by a key life event such as a birthday, are educated and professional, and often disclose their treatment to close friends and family. Understanding the psychological aspects of egg freezing patients will engender clinicians the ability to meet patients' needs and appropriately counsel them.
\end{abstract}

Keywords: Eelective Oocyte Cryopreservation; Basal

${ }^{*}$ Corresponding author.
Antral Follicle Count (BAFC); Psychological

Counseling; Modern Society; Motherhood

\section{INTRODUCTION}

In modern society, women find it increasingly important to achieve personal goals and achievements. Most recently, women aim to complete their education, obtain financial security, stabilize good housing and secure strong relationships before engaging in the responsibility of parenthood [1,2]. With these goals in mind, there has been increased interest in the advent of utilizing fertility preservation (FP) techniques to freeze young oocytes and to reduce the time pressure both on creating the conditions to start a family, and on the actualization in achieving other life plans [3].

Nearly $8 \%$ of children born today are from women older than 35, a trend which is on the rise [4], and more than 1000 children are born worldwide from cryopreserved oocytes [5]. Studies report that from 1990 to 2008, births to unmarried women increased from $28 \%$ to $41 \%$. Of this $41 \%, 14 \%$ corresponded to women $>35$ years [6]. With these available choices, women are able to understand and develop different perspectives and representations of motherhood, and may consider a multitude of options including artificial reproductive techniques (ART) to achieve their goals [7].

The American Society of Reproductive Medicine (ASRM) considers oocyte cryopreservation (OC) experimental. Although it has been shown in several studies to be safe and potential effective [3-8], a number of couples have shown apprehension in pursuing medical treatment or to participate in infertility research [9]. A variety of subjective reasons have surfaced for this including by not limited to treatment duration and cost, an openness to adoption, fear of the medical exploitation and scorn by social-cultural beliefs that do not consider assisted repro- 
ductive techniques (ART) as an alternative to name a few [9]. Such ill feelings require clinicians to fully inform patients about the characteristics of the procedure, includeing the potential of further applications with in vitro fertilization (IVF) or intracytoplasmic sperm injection (ICSI). We consider a comprehensive understanding of the limitations in oocyte preservation must be disclosed to ensure patients understand all perspectives and potential outcomes of such a procedure.

In addition to clinician and technical explanation of OC, ASRM recommends psychological counseling by qualified mental health professional [10]. Mertes H. et al. recommend that the best way to counsel candidate freezers about their reproductive options is to take into consideration the age, family history of premature ovarian failure and ovarian reserve at all ages [1]. Continued evaluations with several blood tests measuring hormone levels and conducting a vaginal ultrasound are suggested.

Complex social influences and psychological impact of oocyte cryopreservation have garnered much discussion and debate. Although there has been much research on this topic as it relates to methodology, there have been no published analyses on the physiological and social aspects of women seeking elective FP. A closer inspection could improve reproductive medicine's understanding as to optimal counseling and education techniques for such patients.

Our study investigated this population by compiling and IRB approved series of surveys over a seven year period. Data were derived from counseling sessions with highly specified mental health professionals along with information derived from an electronic medical data base with regard to patient demographics and outcomes. Our study's goal is to clarify many of these theories and speculations into the psychological and social aspects that are related to women who choose elective OC.

\section{MATERIALS AND METHODS}

We performed an analysis of our internal medical database, focusing on all of our different retrospective studies conducted at a private, academic center (Reproductive Medicine Associates of New York (RMA of NY)) between 2004-2011.

\subsection{Motivational Assessment}

Psychological consultations of 20 patients going through an elective OC cycle (July 15, 2005 and April 15, 2006) were reviewed. Demographic data were retrieved via an electronic medical database. Supplemental data were retrieved from patient's sessions with staff psychologist.

\subsection{Multi-Center National Perspective Analysis}

Female patients $(n=1907)$ were inquired about OC technology at 4 different IVF centers around the country
(Texas Fertility Center, Austin, Tx; Huntington Reproductive Center (HRC), LA, California; IVF New Jersey, Sumerset, NJ and Reproductive Medicine Associates of New York, New York, NY). One hundred and ninetyseven women completed an OC medical consult. After IRB-approved consent, 82 patients underwent controlled ovarian hyperstimulation $(\mathrm{COH})$ for elective $\mathrm{OC}$ using gonadotropin releasing hormone $(\mathrm{GnRH})$ antagonists or GnRH microflare protocol. Baseline screening included transvaginal ultrasound, cycle day 3 follicle stimulating hormone (FSH) and estradiol (E2) levels. Gonadotropins were adjusted based on follicular growth and E2 levels. Final oocyte maturation was achieved using human chorionic gonadotropin with retrieval $36 \mathrm{~h}$ later. A slow freeze protocol including 1,2-propanediol and sucrose was used to freeze oocytes. Sites were compared by age, day $3 \mathrm{FSH}$ and E2 levels, stimulation duration, total gonadotropin dose, peak E2 level, and number of oocytes.

\subsection{Multi-Center Analysis of Age Related to OC Success}

Patient age having interest in fertility preservation and completion of cryopreservation cycle, and success of oocyte cryopreservation were examined retrospectively, in an IRB-approved, multi-center clinical study. Demographic data from women inquiring about fertility preservation by contacting a single call center from January 2004 through July 2007 was collected. All patients went through an informed consent process as per center protocol and signed informed consent forms. Potential patients were followed in a longitudinal fashion. The success of an oocyte cryopreservation cycle was gauged by examining the number of MII oocytes cryopreserved (of cycles that reported maturity). Statistical analysis was performed using t-test for continuous variables and chisquared for non-parametric data.

\subsection{Health Care Providers Recommendation}

A total of 29 surveys were sent out to physicians, nurses, and IVF coordinators of 5 IVF centers in the United States that offer oocyte cryopreservation technology coordinated through a multicenter IRB-approved Extend Fertility study: Reproductive Medicine Associates of New York (RMA), Huntington Reproductive Center (HRC), Texas Fertility Center (TFC), Boston IVF (BIVF) and IVF New Jersey (IVFNJ).

\subsection{Local and National Economy Effect on Elective OC}

Patients $(n=315)$ from the New York City (NYC) area were inquired about oocyte cryopreservation from Nov- 
ember 2004 to March 2009. Patients facing potentially sterilizing cancer treatment were excluded [9]. Economic indicators, including monthly NYC unemployment, the Conference Board Consumer Confidence Index (CCI), and closing values of the Dow Jones Industrial Average (DJIA) were correlated with new patient consults and cycle starts, as were New York County per capita income (PCI) and NYC annual unemployment data.

\subsection{Patient Disclosure}

Age trend, sharing with friends/family and single motherhood were surveyed among women $(\mathrm{n}=230)$ who were consulted for consideration of oocyte cryopreservation between the periods of $7 / 15 / 05$ to $8 / 31 / 11$. The age of the population studies was recorded. All received a counseling session with one of two mental health professionals in a large reproductive medicine office. In this meeting, patients were asked several preset questions where two main focuses were addressed in surveys. We evaluated as to whether patients shared their decision with family and/or friends when they engaged in elective OC. We then inquired patient's perspective of single motherhood in relation to stage of life; and if performing OC would be a viable alternative. Survey and interview responses were recorded and analyzed to assess general disclosure behaviors.

\subsection{Clinical Assessment of Fertility in Women Considering OC}

519 cycles (2005-2010) were analyzed by women who were considering the use of OC. We analyzed age, day 3 follicle stimulating hormone (FSH), basal antral follicle count (BAFC), gonadotropin dose, and the number and stage of oocytes retrieved/cryopreserved. Statistical analysis was performed using Student's t-test and Pearson's correlation.

\section{RESULTS}

\subsection{Motivational Assessment}

Mean patient age was $38.6 \pm 1.83$. All patients had earned a bachelor's degree, and $75 \%$ had received their masters or professional degree. All patients $(n=20)$ were single, although 4 patients $(20 \%)$ were in committed relationships. None had prior full-term pregnancies. With an open-ended question, patients were asked to categorize their motivation: $60 \%(n=12)$ reported that they elected to freeze their eggs to be sure they had taken advantage of all possible reproductive opportunities. $50 \%(\mathrm{n}=10)$ said they felt pressured by their biological clock. $15 \%$ of patients $(n=3)$ said they wanted to freeze eggs as an "insurance policy", but that they would probably never end up using them. When asked their reasons for applying to the program, $65 \%(\mathrm{n}=13)$ said they recently became aware of egg freezing technology; while $20 \%(n=4)$ said they were applying because of their age. $40 \%$ of patients $(n=8)$ said they would definitely be willing to single parent, another $40 \%(n=8)$ said they were unsure if they would be willing to single parent, and the final $20 \%(\mathrm{n}=4)$ said they would not be willing to single parent. Of those that said they would single parent, the average age they wanted to use their eggs was 40.75 (with a range of $40-43$ years). When asked to describe themselves, the most common descriptions were "intelligent" and "outgoing". Other self-descriptors cited were active, warm, focused, considerate, hopeful, happy, intense, adventurous, and determined.

\subsection{Multi-Center National Perspective Analysis}

Mean age of inquiry was $35.2 \pm 5.4$ years. Baseline screening results were similar for all four centers (Texas Fertility Center, Austin, Tx; Huntington Reproductive Center (HRC), LA, California; IVF New Jersey, Sumerset, NJ and Reproductive Medicine Associates of New York, New York, NY). No significant differences were encountered for age (mean 38.01 \pm 2.26 ), day three FSH levels (mean $8 \pm 3.3 \mathrm{IU} / \mathrm{L})$ or day 3 E2 levels $(39.8 \pm$ $14.1 \mathrm{pg} / \mathrm{ml})$. The mean length of stimulation was $10 \pm$ 1.6 days. TFC patient's length of stimulation was significantly longer in comparison to the other centers ( $\mathrm{P}$ $<0.0123$ ). A trend towards a lower peak E2 was noted in patients from RMANY $(\mathrm{p}=0.0745)$. The total dose of gonadotropins (mean $4135 \pm 1324 \mathrm{IU}$ ), number of retrieved oocytes $(12.7 \pm 6.8)$ and MII oocytes $(9.6 \pm 6.3)$ were similar among groups (Table 1).

\subsection{Health Care Providers Recommendation}

A total of 18 completed surveys were returned: 13 from physicians and 5 from registered nurses and IVF coordinators. When asked about the ideal age at which a woman should undergo an oocyte cryopreservation procedure, $56.3 \%$ considered the best age to be $<35$, followed by 28 - 35 years $(18.75 \%)$ and 25 - 28 years $(12.5 \%)$, and the mean upper limit age accepted for this technique was 39.9 - 1.4 years. Fifty percent of providers considered it acceptable for a woman to undergo this procedure with a day $3 \mathrm{FSH}<13 \mathrm{IU} / \mathrm{L}$. When the cut-off level was changed to $<18 \mathrm{IU} / \mathrm{L}, 100 \%$ of the providers rejected these women as candidates to undergo the procedure. Seventy-eight percent of those surveyed would recommend against offering the procedure to patients with a BAFC of $<5$. When asked about whether their recommendation to undergo oocyte cryopreservation would change if it were done for medical rather than elective indications, $89 \%$ of providers answered yes. 
Table 1. Multi-center national perspective analysis.

\begin{tabular}{|c|c|c|c|c|c|}
\hline & IVF NJ $n=10$ & $\mathrm{TFC} \mathrm{n}=11$ & $\mathrm{HRC} \mathrm{n}=14$ & RMANY n $=47$ & Overall \\
\hline Age & $37.0 \pm 3.0$ & $37.5 \pm 2.1$ & $37.4 \pm 2.5$ & $38.5 \pm 2.0$ & $38 \pm 2.3$ \\
\hline FSH (Day 3) & $7.2 \pm 3.6$ & $7.1 \pm 1.2$ & $7.0 \pm 1.9$ & $8.7 \pm 3.8$ & $7.9 \pm 3.2$ \\
\hline E2 (Day 3) & $33.9 \pm 9.5$ & $42.6 \pm 15.5$ & $40.0 \pm 17.9$ & $40.6 \pm 12.1$ & $39.9 \pm 14.1$ \\
\hline Days Stim & $9.3 \pm 1.3^{*}$ & $11.5 \pm 1.4^{*}$ & $9.9 \pm 1.8^{*}$ & $9.9 \pm 1.5^{*}$ & $10.05 \pm 1.6$ \\
\hline Total GND Dose & $3652 \pm 1102$ & $3731 \pm 2010$ & $4441 \pm 1670$ & $4245 \pm 1010$ & $4135 \pm 1324$ \\
\hline Peak E2 & $2698 \pm 1188$ & $2379 \pm 743$ & $2323 \pm 1269$ & $1897 \pm 1172$ & $2148 \pm 1157$ \\
\hline \#Ret Oocyte & $13.4 \pm 7.4$ & $15.8 \pm 4.4$ & $9.9 \pm 4.8$ & $12.3 \pm 7.5$ & $12.7 \pm 6.8$ \\
\hline \#MII & $11.7 \pm 6.8$ & $11.9 \pm 4.4$ & $9.0 \pm 4.8$ & $8.6 \pm 6.8$ & $9.6 \pm 6.3$ \\
\hline
\end{tabular}

Note: Follicle Stimulating Hormone: FSH; Estradiol: E2; Days of Stimulation: Days Stim; Gonadotropin: GND; Number of Retrieved Oocytes: \#Ret Oocyte; Number of Metaphase II Oocytes: \#MII.

The take-home baby rate, estimated by providers, for women $<35$ years old was $38.1 \% /$ ET $(n=13)$. The average live birth rate for women $>35$ years old was thought to be $24.8 \% / \mathrm{ET}(\mathrm{n}=12)$. A limited number of respondents estimated that it would take $\sim 24$ frozen oocytes for each take-home baby in women $<35$ and $\sim 40$ oocytes for those $>35$. The providers were asked about what the future holds for this procedure in the next 5 years, and 16 agreed that oocyte cryopreservation will be used more than now and only 1 person did not provide an answer. They were questioned about what the prevailing technique will be in 5 years: $33 \%(n=6)$ thought that the slow-freezing technique would predominate, and 39\% (n $=7$ ) believed that it would be vitrification. The remaining $28 \%$ did not provide an answer. Approximately 50\% of those surveyed accepted that in vitro maturation and/or orally active gonadotropins would decrease the cost of this procedure. When providers were asked about their recommendations for a patient, who while inquiring about fertility preservation received lab tests that suggested a pre-existing diminished ovarian reserve; nearly half recommend that the patient change her plans and attempt conception as soon as possible. The most common concerns expressed providers by women, presenting for elective oocyte cryopreservation, were outcome of the technique and ovarian aging $(61 \%)$, followed by social aspects of their lives and, finally, cost and safety. Eighty-seven percent of the providers address these concerns by thorough discussion and current data presentation.

\subsection{Local and National Economy Effect on Elective OC}

Correlation was noted between new consults and per capita annual income $(\mathrm{r}=0.98, \mathrm{p}=0.02)$ and annual unemployment $(\mathrm{r}=-0.91, \mathrm{p}=0.03)$. Monthly economic variables did not correlate with new patient appointments: unemployment $(\mathrm{r}=-0.17, \mathrm{p}=20.2)$, DJI $(\mathrm{r}=0.20, \mathrm{p}=$ $0.14)$, CCI $(\mathrm{r}=-0.23, \mathrm{p}=0.09)$; or with monthly cycle starts: unemployment $(\mathrm{r}=0.01, \mathrm{p}=0.94)$, DJI $(\mathrm{r}=0.19$, $\mathrm{p}=0.21)$, and CCI $(\mathrm{r}=0.09, \mathrm{p}=0.55)$.

\subsection{Age Trend of Surveyed Patients}

The mean age of women $(n=230)$ considering OC in 2005 was $39+1.4$ years and diminished to $37.4+2.3$ years in 2011. Pearson Correlation showed age trending downward but was not statistically significant (r-value = 0.651, $\mathrm{p}=0.113$ ) (Table 2).

\subsection{Disclosure Trends}

Of these women $(\mathrm{n}=230)$ considering OC, $79 \%(\mathrm{n}=$ 181) reported discussing OC with "friends", 86\% ( $\mathrm{n}=$ 197) reported discussing their treatment with "family" and $64 \%(n=148)$ reported discussions with both friends and family. Four patients did not disclose with any of the groups (1\%) (Figure 1).

\subsection{Single Motherhood}

In interviews that asked whether patients contemplated single motherhood with their frozen oocytes, younger women responded "yes" more often to this question. Of women age $\leq 37(\mathrm{n}=97), 62 \%(\mathrm{n}=60)$ responded "yes"; while $54 \%(\mathrm{n}=72)$ of women age $\geq 38(\mathrm{n}=133)$ responded "no". Of all patients $(\mathrm{n}=230), 15 \%(\mathrm{n}=35)$ reported they were not sure if $\mathrm{OC}$ was a viable option and expressed that they would have to consider that potential upon the present time circumstances (Figure 2).

\subsection{Fertility Assessment in Women Considering OC}

In a population analyzed presenting for new patient consultations $(\mathrm{n}=519), 178$ patients initiated a total of 236 
Table 2. Age trend of surveyed patients considering oocyte cryopreservation.

\begin{tabular}{cccccccc}
\hline & 2005 & 2006 & 2007 & 2008 & 2009 & 2010 & 2011 \\
\hline Mean age \pm SD & $39 \pm 1.4$ & $38.1 \pm 1.9$ & $37.4 \pm 2.4$ & $37.4 \pm 2.5$ & $37.9 \pm 2.1$ & $37.9 \pm 2.4$ & $37.4 \pm 2.3$ \\
\hline
\end{tabular}

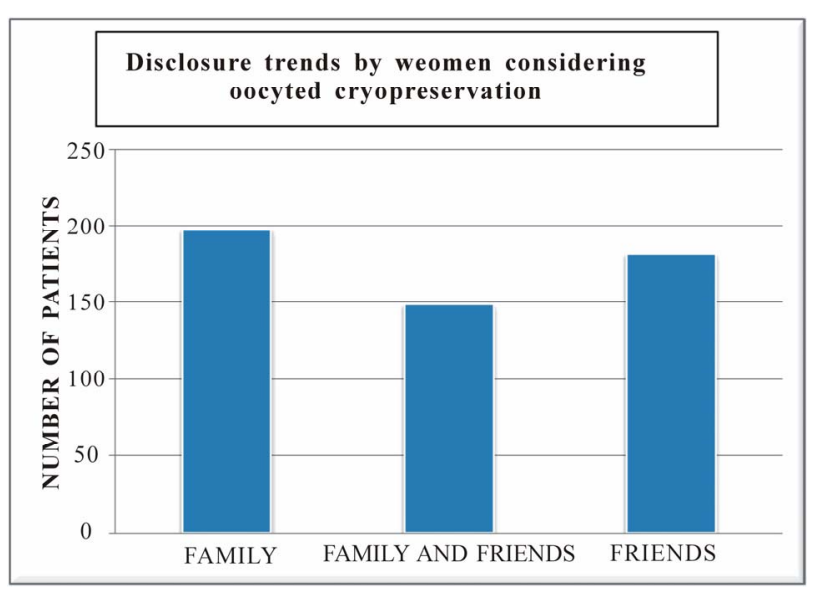

Figure 1. Disclosure trends by women considering oocyted cryopreservation.

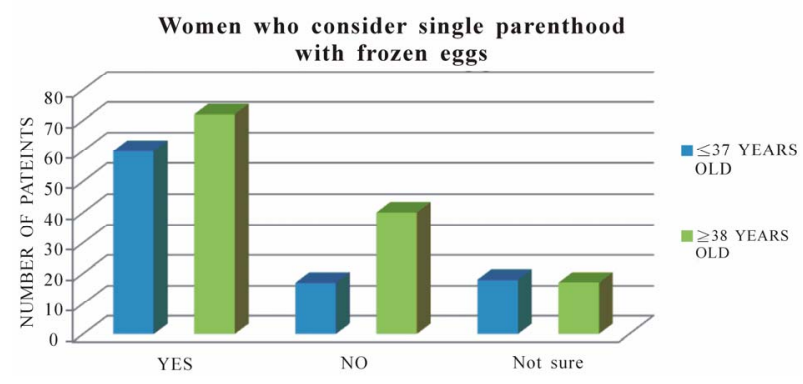

Figure 2. Women who consider single parenthood with frozen eggs.

OC cycles. Twenty-four cycles were cancelled (9.6\%) due to poor ovarian response or self-withdrawal after initiating controlled ovarian hyperstimulation. Although there was no statistical difference in patients age, those with a cancelled cycle had a higher FSH $13+7$ IU/1 $(\mathrm{p}<$ $0.02)$ and lower BAFC $6+2(\mathrm{p}<0.02)$ than the patients with completed cycles. In 212 completed cycles, the mean number of mature OC was $13.5+8.8$. Indicators (in order of importance) most correlated to number of OC were BAFC, FSH and age (all p < 0.0.5) (Table 3).

\section{DISCUSSION}

As reproductive medicine celebrates the recent 50th anniversary of the introduction of "the pill," society has come to recognize that women have successfully controlled their reproduction for more than five decades. Indeed, reproductive freedoms have been a stepping-stone for broader female emancipation [11]. Society has also observed a related delay in childbearing in most
Table 3. Fertility assessment in women considering oocyte cryopreservation.

\begin{tabular}{ccccc}
\hline Age Group & $<35$ & $35-37$ & $38-40$ & $>40$ \\
Cycles & 24 & 69 & 92 & 26 \\
Day 3 FSH & $8.1 \pm 5.2$ & $8.9 \pm 3.0$ & $9.7 \pm 4.5$ & $8.7 \pm 2.9$ \\
BAFC & $10.3 \pm 5.4$ & $9.1 \pm 3.7$ & $8.0 \pm 4.1$ & $7.8 \pm 4.8$ \\
MII Cryo & $10 \pm 7.2$ & $9.6 \pm 6.4$ & $9.0 \pm 7.7$ & $5.0 \pm 3.3$ \\
\hline
\end{tabular}

part of Europe and with high-educated women in the United States [11,12]. From a social anthropological vantage point, over the past seven years we have studied the introduction and incorporation of oocyte cryopreservation technology into a population of reproducetive age women. After reviewing the literature, we did not find any similar studies evaluating a cohort of women presenting for elective egg freezing. Our findings sheds light on this population, creates a better understanding of motivating factors, demographic trends, and realistic expectations, and adds to our understanding of social and demographic trends.

\subsection{Age Trends}

It has been reported that young women often are unaware of the early decline of their fertility; they postpone childbearing during their most fertile years expecting to conceive as soon as they try [6]. We observed this perception within patients undergoing OC. Although women mean age of inquiring about this procedure is $35.2 \pm 5.4$ years, in our studies, the actual mean age of women undergoing OC was $37.87 \pm 2.1(\mathrm{n}=230)$ and $38.7+1.8(\mathrm{n}$ $=519$ ), respectively, over a period of 5 years (January 2005-December 2010) demonstrating statistical significance $(\mathrm{p}<0.01)$. When we compared this data to the national perspective we encountered no significant difference in mean age $(38.01 \pm 2.26)$. These data points are comparable with previous research $[1,2]$, and women at such ages have been shown to lead to a lower successful pregnancy rate. Our study supports the necessity for early availability of counseling and education on fertility preservation. Of the health care providers surveyed, $56.3 \%$ suggested that this education be offered prior to the age of 35 , as this age is considered to be the limit marker generally used for oocyte donor recruitment. They also estimate that the take-home baby rate for women $<35$ was $38.1 \%$ compared to $24.8 \%$ in women aged $>35$. In addition, early edification could assuage 
genetic abnormality such as aneuploidy, which has been shown in previous research to be between $30 \%-45 \%$ in human zygotes in women $\leq 34$, and could double as a woman nears $\geq 38$ years $[2,13]$. These findings could relate to the fact that women $>35$ need $\sim 40$ oocytes for each take-home baby and have a $15 \%$ less chance of cryopreserving more than 10 MII oocytes per cycle in contrast to younger women $(<35)$.

\subsection{Single Motherhood}

A previous study conducted by Stoop et al. (2010) investigated the social background behind elective OC. This study performed a survey in Belgium of 1914 women between the ages of 19 - 40 years and reported that $3.1 \%$ of women accepted the idea of cryopreservation, $28.4 \%$ considered the possibility and $51.8 \%$ were opposed. The analysis showed that those in favor were younger than the rest of the population [2]. Similar data was obtained in our analysis; younger patients $(<37$ years old) seeking elective cryopreservation, were more prone to the idea of becoming a single parent than patients $\geq 38$ years $(63 \%$ vs. 54\% respectively). This was an increase from 2006 , when only $40 \%$ of our patients say they would definitely pursue single motherhood and another $40 \%$ were unsure; a finding that attributes to the evolution turn in reproduction demographics.

\subsection{Disclosure Friends/Family}

A previous study reported that the severity in diagnosis realization of infertility caused psychological morbidity; such as a reduction in one's sense of well-being and self-determination over reproduction [14]. Patients felt less comfortable discussing the situation and even avoided conversation for alternatives with other people [14]. This was thought to be in part, due to the perception or real social unacceptability and lack of empathy from family and friends regarding infertility [9]. On the contrary, our study demonstrated a change in that view. Patients who pursued non-medical OC disclosed the procedure, and were more open about this decision with family and friends, suggesting that it may be an accommodating and empowering action. There was a slightly difference in the number of patients that preferred sharing with family other than friends (83\% vs. $79 \%$ respectively); and 65\% (157 patients) answered that they shared with both groups.

\subsection{Fertility Assessment}

On a clinical standpoint, we have reinforced a women's age is the chief factor that serves as a biological hallmark related with the ovarian reserve. Both are a fundamental aspect on the evaluation of woman's fertility and a guideline for the most appropriate management. The most efficient way to evaluate the ovarian reserve, is measuring FSH on day 3 of the menstrual cycle. Levels $>$ $15 \mathrm{mIU} / \mathrm{ml}$ suggest decreased ovarian reserve and a reduced probability of pregnancy [15]. The latest marker used is the anti-Müllerian hormone (AMH), where levels $>1 \mathrm{ng} / \mathrm{ml}$ are associated to poor ovarian response. Such diagnostic tools could be used by younger women, such as in there early and mid-20s, especially with those whose family history trends of premature ovarian failure as such preventive measures would provide the opportunity for conception at a more suitable time. In addition to these diagnostic tools, our study demonstrated further clinical associations with statistical significance $(p<$ 0.001 ) to OC with the following factors: the number of oocytes retrieved, cryopreservation of mature oocytes (MII), and the measurement basal antral follicular count (BAFC) during a vaginal ultrasound; correlations to the national perspective and with the health care provider's recommendation. The majority of $50 \%$ of physicians surveyed considered acceptable day $3 \mathrm{FSH}$ level to be $<13 \mathrm{mlU} / \mathrm{ml}$ and $78 \%$ were against performing the procedure with a BAFC of $<5$. There was no significant difference when we compare our 5-year mean number of oocytes retrieved and the mature oocyte count (MII) to the national perspective $(13.5 \pm 8.8$ vs. $12.7 \pm 6.8$ and 8.4 \pm 4.4 vs. $9.6 \pm 6.3$ respectively).

\subsection{Motivation and Concerns}

Advances in technology and public awareness have served to form the impetus for patients to consider and possibly undergo oocyte cryopreservation. A number of women voiced they were interested in egg freezing to take the pressure off the search for relationships, since cryopreservation for them meant the freedom to wait, avoiding feelings of pressure to settle for a mate due to social perceptions on the appropriate time for females to conceive and ultimately raise a family. More than half of the patients $(61 \%)$ were concerned about the outcome of the technique, ovarian aging and the social aspects of their lives; such as in costs and safety. Stoop et al, reported if potential freezers are reassured about risks to their future fertility related to the procedure, $75.2 \%$ of the potential freezers would embark on elective OC [16]. Further research is necessary to establish if ovarian stimulation and oocyte retrieval affect the future fertility in healthy women.

\subsection{Economy Effect on Elective OC}

Indicators such as industrial output, wealth and consumer sentiment were found not to be deterrents for a patient consultation, albeit per capita income and annual unemployment data were found to restrain patient appeal. A study made in Belgium found a trend regarding monthly 
income with non-freezers where patient with a "medium income" were less interested in OC than a "low income" patients $\left[\mathrm{x}^{2}(4)=7.96, \mathrm{p}<0.01\right]$ [16]. Importantly, the Conference Board Consumer Confidence Index, did not correlate income with appointments for OC, suggesting that patients desiring egg freezing may not be derailed by short-term sentiment [16]. With these findings we consider patients will pursue elective OC if they perceive it as a time-dependent, necessary medical procedure, even during times of economic stress.

Although patients are thoroughly counseled regarding realistic expectations of egg freezing and are aware that oocytes may be chromosomal abnormal, and may not always freeze, thaw, or implant with a high efficiency, women who freeze oocytes are empowered and report being quite optimistic about their possibility of conceiving at a later stage of life. Freezing oocytes at a younger age can mitigate the deleterious effects of age (e.g. aneuploidy, miscarriage rate), potentially provide the same quality as a fresh oocyte $[3,4]$, improve the outcome for a successful pregnancy, and enhances the overall quality of life. Women become less pressured by time to find a life partner or they may consider single parenthood as an alternative, by using cryopreserved younger oocytes. With our comprehensive study and effective counseling by our mental health professionals, it appears that elective $\mathrm{OC}$ is increasingly being pursued, becoming a highly accepted procedure by women at younger ages and based on the fact that women are sharing with friends and family that they are going through treatment, it appears that social acceptance of elective oocyte cryopreservation is increasing.

\section{REFERENCES}

[1] Mertes, H. and Pennings, G. (2011) Social egg freezing: For better, not for worse. Reproductive BioMedicine Online, 23, 824-829. doi:10.1016/j.rbmo.2011.09.010

[2] Soliman, H.H., Khaki, A.A., Al-Azawi, T. and Al-Harani, S. (2012) Oocyte cryopreservation, will it be a real social choice and family solution? Middle East Fertility Society Journal, 17, 8-11. doi:10.1016/j.mefs.2012.01.003

[3] Dondorp, W., de Wert, G., Pennings, G., Shenfield, F., Devroey, P., et al. (2012) Oocyte cryopreservation for age-related fertility loss. Human Reproduction, 27, 12311237. doi:10.1093/humrep/des029

[4] Goold, I. and Savulescu, J. (2009) In favor of freezing eggs for non-medical reasons. Bioethics, 23, 47-58. doi:10.1111/j.1467-8519.2008.00679.x
[5] Rodriguez-Wallberg, K. and Oktay, K. (2012) Recent advances in oocyte and ovarian tissue cryopreservation and transplantation. Best Practice \& Research Clinical Obstetrics and Gynaecology, 26, 391-405. doi:10.1016/j.bpobgyn.2012.01.001

[6] Dondorp, W.J. and De Wert, G.M.W.R. (2009) Fertility preservation for healthy women: Ethical aspects. Human Reproduction, 24, 1779-1785.

[7] Hirshfeld, J., Grobman, W. and Milad, M.P. (2012) Fertility preservation for social indications: A cost-based decision analysis. Fertility and Sterility, 97, 665-670. doi:10.1016/j.fertnstert.2011.12.029

[8] Dondorp, W., et al. (2012) Oocyte cryopreservation for age-related fertility loss. Human Reproduction, 27, 12311237. doi:10.1093/humrep/des029

[9] Cousineau, T.M. and Domar, A.D. (2007) Psychological impact of infertility. Best Practice \& Research Clinical Obstetrics and Gynecology, 21, 293-308. doi:10.1016/j.bpobgyn.2006.12.003

[10] (2008) Essential elements of informed consent for elective oocyte. The Practice Committee of the Society for Assisted Reproductive Technology and the Practice Committee of the American Society for Reproductive Medicine.

[11] Mills, M., Rindfuss, R., McDonald, P. and Te Velde, E. (2011) Why do people postpone parenthood? Reasons and social policy incentives. Human Reproduction, 17, 848-860. doi:10.1093/humupd/dmr026

[12] Dhont, M. (2010) History of oral contraception. The European Journal of Contraception and Reproductive Health Care, 15, S12-S18. doi:10.3109/13625187.2010.513071

[13] Kuliev, A., Cieslak, J. and Verlinsky, Y. (2005) Frequency and distribution of chromosome abnormalities in human oocytes. Cytogenetic and Genome Research, 111, 193-198. doi:10.1159/000086889

[14] Dudzinski, D. (2004) Ethical issues in fertility preservation for adolescent cancer survivors: oocyte and ovarian tissue cryopreservation. Journal of Pediatric and Adolescent Gynecology, 17, 97-102. doi:10.1016/j.jpag.2004.01.004

[15] Marhhom, E. and Cohen, I. (2007) Fertility preservation options for women with malignancies. Obtetrical and Gynecological Survey, 62, 58-72. doi:10.1097/01.ogx.0000251029.93792.5d

[16] Stoop, D., Nekkebroeck, J. and Devroey, P. (2011) A survey on the intentions and attitudes towards oocyte cryopreservation for non-medical reasons among women of reproductive age. Human Reproduction, 26, 655-661. doi:10.1093/humrep/deq367 